\title{
Lung metastases in low-risk gestational trophoblastic neoplasia: a retrospective cohort study
}

\author{
MM Frijstein, ${ }^{a, b, c}$ CAR Lok, ${ }^{a}$ NE van Trommel, ${ }^{a}$ MJ ten Kate-Booij, ${ }^{b}$ LFAG Massuger, ${ }^{c}$ \\ E van Werkhoven, ${ }^{d}$ D Short, ${ }^{e}$ X Aguiar, ${ }^{e}$ RA Fisher, ${ }^{e}$ B Kaur, $^{e}$ N Sarwar, ${ }^{e}$ NJ Sebire, ${ }^{e}$ MJ Seckl ${ }^{e}$ \\ ${ }^{a}$ Department of Gynaecological Oncology, Centre of Gynaecological Oncology Amsterdam, Amsterdam, the Netherlands ${ }^{\mathrm{b}}$ Department of \\ Obstetrics and Gynaecology, Erasmus University Medical Centre, Rotterdam, the Netherlands ' Department of Obstetrics and Gynaecology, \\ Radboud University Medical Centre, Nijmegen, the Netherlands ${ }^{\mathrm{d}}$ Department of Biometrics, Netherlands Cancer Institute - Antoni van \\ Leeuwenhoek, Amsterdam, the Netherlands ${ }^{\mathrm{e}}$ Department of Medical Oncology, Charing Cross Hospital, London, UK \\ Correspondence: MM Frijstein, Department of Gynaecological Oncology, Centre of Gynaecological Oncology Amsterdam, Location Netherlands \\ Cancer Institute - Antoni van Leeuwenhoek Hospital, Plesmanlaan 121, 1066 CX Amsterdam, The Netherlands. Email: m.frijstein@nki.nl
}

Accepted 29 November 2019. Published Online 20 December 2019.

\begin{abstract}
Objective Presence of lung metastases in low-risk gestational trophoblastic neoplasia (GTN) is generally considered not to influence prognosis. However, in a recent study in the Netherlands, GTN patients with lung metastases had a higher recurrence rate and more disease-specific deaths compared with patients without metastases. The aim of the present study was to validate these findings in a different country.
\end{abstract}

Design Historical cohort study.

Setting Charing Cross Hospital, United Kingdom.

Population A total of 1040 low-risk GTN patients treated with methotrexate (MTX) between 2002 and 2016 were identified: 65 with lung metastases (group 1) and 975 without metastases (group 2).

Methods Baseline characteristics, MTX resistance, survival and recurrence rates were recorded and compared between both groups.

Main outcome measures MTX resistance, recurrence rate and survival.

Results The occurrence of MTX resistance and median number of MTX courses to achieve remission was significantly higher in patients with lung metastases than patients without metastases ( $60 \%$ versus $38.9 \%, P=0.001$; and nine versus six courses, $P<0.001)$. All choriocarcinoma patients $(n=4)$ with lung metastases developed MTX resistance. The recurrence rate was also higher in group I $(9.2 \%$ versus $2.7 \% ; P=0.012)$. Diseasespecific survival was $100 \%$ in both groups.

Conclusions The presence of lung metastases at the start of MTX therapy is associated with increased incidence of MTX resistance and recurrence in low-risk GTN without affecting overall survival, which remains $100 \%$. However, individuals with low-risk choriocarcinoma with lung metastases are likely to become resistant to MTX and primary multi-agent chemotherapy should be considered.

Keywords Gestational trophoblastic neoplasia, lung metastases, methotrexate, recurrence.

Tweetable abstract The presence of lung metastases appears to increase the risk of recurrence in low-risk GTN, but does not affect overall cure rates and survival.

Linked article This article is commented on by PFW Chien, p. 396 in this issue. To view this mini commentary visit https://doi.org/10.1111/1471-0528.16035.

Please cite this paper as: Frijstein MM, Lok CAR, van Trommel NE, ten Kate-Booij MJ, Massuger LFAG, van Werkhoven E, Short D, Aguiar X, Fisher RA, Kaur B, Sarwar N, Sebire NJ, Seckl MJ. Lung metastases in low-risk gestational trophoblastic neoplasia: a retrospective cohort study. BJOG 2020;127: 389-395.

\section{Introduction}

Gestational trophoblastic neoplasia (GTN) is a relatively rare malignant condition that comprises entities such as: persistently increased human chorionic gonadotrophin (hCG) after molar pregnancy, non-molar choriocarcinoma, placental site trophoblastic and epithelioid trophoblastic tumours (PSTT/ETT). ${ }^{1}$ Treatments have evolved so that GTN is now a disease with high cure rates even in the presence of metastatic disease. ${ }^{2-5}$ According to the criteria of the International Federation of Gynecology and Obstetrics (FIGO), post-molar GTN is diagnosed when one of the 
following is present: (1) a plateau of at least four persistently elevated hCG values during 3 weeks, (2) a sequential rise of three hCG values for 2 weeks or longer, (3) histological diagnosis of choriocarcinoma., ${ }^{2,6}$ GTN is treated with either single-agent or multi-agent chemotherapy, which in nearly all parts of the world is selected using the FIGO 2000 scoring system. This enables the stratification of individuals based on whether they have a low or high risk of developing resistance to single-agent chemotherapy. ${ }^{6,7}$ In the Netherlands, a clinical scoring system was used until recently. ${ }^{8,9}$ In both the FIGO and Dutch clinical scoring systems, the lung as a site of metastases is not considered to be an adverse prognostic factor. However, the total number of metastases and the maximum tumour size, which includes metastases in the lungs, can influence the FIGO score and thereby impact on the selection of singleagent versus multi-agent chemotherapy. A chest X-ray (CXR) is recommended to assess lung metastases to evaluate the risk score, and not chest computed tomography (CT). ${ }^{2}$ In the recent study by Vree et al., patients with lung metastases had a higher pre-evacuation level of hCG, more recurrences and a higher chance of dying from their disease. ${ }^{9}$ As this study was performed using the Dutch scoring system in a setting where the care of trophoblast patients is not centralised in expert centres, this could have resulted in a worse outcome with disease-specific deaths. Therefore, we investigated the influence of lung metastases on the course of disease in a centralised population treated in a specialised trophoblastic disease centre that uses the FIGO scoring system.

\section{Patients and methods}

In this retrospective cohort, all low-risk GTN patients treated initially with methotrexate (MTX) with either no metastases or with lung metastases only, were selected from the electronic database at the Charing Cross Hospital between January 2002 and December 2016.

Figure 1 illustrates the patient selection process. No patients with metastases elsewhere were included. All patients had centralised pathological review by expert pathologists. Patients with PSTT, ETT, mixed PSTT/ETT, mixed PSTT/choriocarcinoma or mixed ETT/choriocarcinoma were excluded from this study because these disease entities behave and are treated differently. ${ }^{10,11}$ High-risk patients (FIGO score $\geq 7$ ) or relapsed low-risk patients following initial chemotherapy were excluded as they are also treated differently. ${ }^{12}$ In addition, 35 low-risk patients treated with first-line therapy other than MTX were excluded to keep the group as homogeneous as possible. All patients were divided into two groups: those with only lung metastases (group 1) and patients without any metastases (group 2). Those with lung metastases were included if their disease was present on initial staging CXR and/or chest CT, without other metastases at any time during the course of the disease. For the definition of GTN, the criteria of FIGO 2000 staging were used. $^{13}$ After diagnosing post-molar GTN, a Doppler ultrasound scan of the pelvis and CXR were performed. If the CXR was abnormal, then a chest CT and an MRI of the brain were undertaken. However, GTN following any other type of pregnancy was investigated with CT of chest/abdomen and MRI of head and pelvis, all with contrast, in addition to a Doppler ultrasound scan of the pelvis. Women scoring $\leq 6$ on the FIGO scoring system were treated with single-agent MTX and folinic acid (FA) chemotherapy as previously described. ${ }^{14,15}$. Patient data were collected on age, antecedent pregnancy, FIGO score, pre-evacuation serum hCG concentrations, pre-chemotherapy serum hCG concentrations, number of lung metastases, the need for single- and multi-agent chemotherapy and the number of courses necessary to achieve normalisation of hCG concentrations. MTX resistance was defined as at least three static or at least two rising serum hCG measurements. ${ }^{15}$ Recurrence was defined as at least two serial rising serum hCG measurements in the absence of a new pregnancy or other explanation after at least 6 weeks of normal hCG values following completion of chemotherapy to induce the first remission of disease. MTX resistance, recurrence and death from disease were recorded.

SPSS software (version 20) and R (version 3.5.0) were used for statistical analysis. Antecedent pregnancies and histopathological diagnosis were compared between patients with and without lung metastases using Fisher exact test. Differences in pre-treatment hCG concentrations, days from evacuation to remission and number of required chemotherapy regimens in patients cured with MTX were calculated and compared between the patients with and without lung metastases using Mann-Whitney $U$ test. Choice of first treatment and effectiveness of MTX treatment, FIGO classification, recurrence of disease and disease-specific survival were compared between both

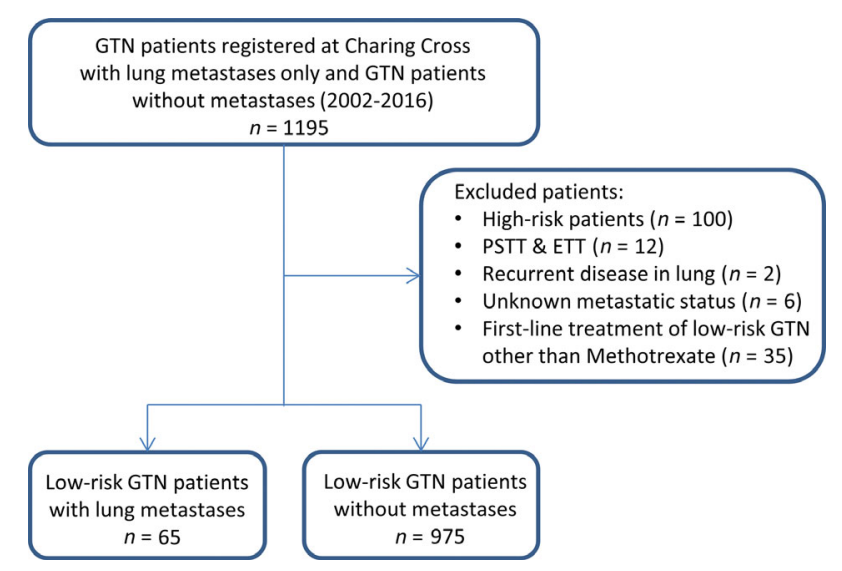

Figure 1. Flow chart illustrating the patient selection process 
patient groups using Fisher exact test. Logistic regression was used to assess the association between presence of lung metastases and recurrence of disease (yes or no) and MTX resistance (yes or no). Because the FIGO scoring system is based on the values assigned to the different prognostic factors, FIGO score was used only in the univariate analysis. Weighted logistic regression was used to estimate the causal effect of the presence of lung metastases. This presence was modelled with antecedent pregnancy, histopathological diagnosis and interval from evacuation until treatment as explanatory variables. Recurrence was analysed as a binary (yes/no) outcome and crude percentages were reported. The results from this method are valid under the assumption that all patients have complete follow up.

No core outcome sets were relevant for this study. Furthermore, there was no patient involvement in this retrospective study and no funding was received.

\section{Results}

A total of 65 low-risk GTN patients with lung metastases (Group I) and 975 without metastases (Group II) were included (Fig. 1). Patient characteristics are presented in
Table 1. A histological diagnosis of choriocarcinoma was more often seen in the patients with lung metastases $(6.2 \%$ versus $1.7 \% P=0.037)$. In contrast, complete molar pregnancies were more often associated with the absence of lung metastasis ( $81 \%$ versus $67.7 \%, P=0.015)$. Low-risk patients with lung metastases had significantly higher preevacuation serum hCG concentrations than patients without metastases (median $85701.50 \mathrm{IU} / 1$ versus median $9654 \mathrm{IU} / \mathrm{l} ; P<0.001$ ), but the difference in pre-chemotherapy serum hCG concentrations was not significant. Age, antecedent pregnancy and the time interval from evacuation to start of therapy did not differ between groups.

In patients with lung metastases, most had only one to four lung lesions $(n=49)$, six had five to eight lesions and only two had more than eight metastases. In eight patients, the available reports simply stated the presence of 'multiple metastases', but further validation was not possible because these images were not accessible for review. The median diameter of the largest lung tumour was $1.3 \mathrm{~cm}$ (range $0.6-4 \mathrm{~cm}$ ). Eight patients had lung metastases only detected by chest CT and not visible on CXR.

Treatment regimen and outcome are presented in Table 2. Patients with lung metastases needed significantly

Table 1. Baseline characteristics of the participants

\begin{tabular}{|c|c|c|c|}
\hline & $\begin{array}{l}\text { Low-risk GTN with lung } \\
\text { metastases (Group I) } \\
\qquad N=65\end{array}$ & $\begin{array}{l}\text { Low-risk GTN without } \\
\text { metastases (Group II) } \\
\qquad N=975\end{array}$ & $P$ value \\
\hline Age in years mean (SD) & $31.5(9.7)$ & $32.4(7.8)$ & 0.366 \\
\hline \multicolumn{4}{|l|}{ Antecedent pregnancy } \\
\hline Mole* & $58(89.2 \%)$ & $926(95.0 \%)$ & 0.106 \\
\hline Term & $2(3.1 \%)$ & $15(1.5 \%)$ & \\
\hline Miscarriage & $5(7.7 \%)$ & $30(3.1 \%)$ & \\
\hline Unknown & $0(0.0 \%)$ & $4(0.4 \%)$ & \\
\hline \multicolumn{4}{|l|}{ FIGO score } \\
\hline Median (IQR) & $4(3-5)$ & $3(1-4)$ & $<0.001$ \\
\hline \multicolumn{4}{|c|}{ Interval from evacuation to start of treatment (months) } \\
\hline Median (IQR) & $1(1-2)$ & $1(1-2)$ & 0.631 \\
\hline \multicolumn{4}{|l|}{ Histopathological diagnosis } \\
\hline Complete mole & $44(67.7 \%)$ & $790(81.0 \%)$ & 0.003 \\
\hline Partial mole & $6(9.2 \%)$ & $109(11.2 \%)$ & \\
\hline Mole* & $7(10.8 \%)$ & $37(3.8 \%)$ & \\
\hline Choriocarcinoma & $4(6.2 \%)$ & $17(1.7 \%)$ & \\
\hline Hydropic abortion & $1(1.5 \%)$ & $5(0.5 \%)$ & \\
\hline Unknown & $3(4.6 \%)$ & $17(1.7 \%)$ & \\
\hline hCG pre-evacuation IU/I & $N=14$ & $N=645$ & \\
\hline Median (range) & $85701.5(492-1165760)$ & $9654(6-1217592)$ & $<0.001$ \\
\hline hCG pre-chemotherapy IU/I & $N=65$ & $N=330$ & \\
\hline Median (range) & 14976 (50-113 533) & $11493.5(1-410804)$ & 0.169 \\
\hline Hysterectomy** & $0(0 \%)$ & $4(0.4 \%)$ & 1.000 \\
\hline
\end{tabular}


Frijstein et al.

Table 2. Outcomes of treatment with MTX in patients with low-risk GTN

\begin{tabular}{lccc}
\hline & $\begin{array}{c}\text { Low-risk GTN } \\
\text { with lung } \\
\text { metastases } \\
\text { (Group I) } \\
\mathbf{N}=\mathbf{6 5}\end{array}$ & $\begin{array}{c}\text { Low-risk GTN } \\
\text { without } \\
\text { metastases } \\
\text { (Group II) } \\
\mathbf{N = 9 7 5}\end{array}$ & $\begin{array}{c}\boldsymbol{P} \text { - } \\
\text { value }\end{array}$ \\
\hline $\begin{array}{l}\text { Median number of } \\
\text { MTX courses to }\end{array}$ & $9(7-10.25)$ & $6(4-7)$ & $<0.001$ \\
$\begin{array}{l}\text { achieve remission, } \\
\text { median (IQR) }\end{array}$ & & & \\
$\begin{array}{l}\text { MTX resistance } \\
\text { Recurrence }\end{array}$ & $39 / 65(60 \%)$ & $379 / 975(38.9 \%)$ & 0.001 \\
Death of disease & $6(9.2 \%)$ & $26(2.7 \%)$ & 0.012 \\
\hline IQR, interquartile range. & $0(0 \%)$ & $0(0 \%)$ & - \\
\hline
\end{tabular}

more MTX courses to achieve remission than patients without metastases (median: nine courses in group I versus six courses in group II). The patients with lung metastases developed MTX resistance more frequently (60\% versus $38.9 \% ; P=0.001)$, but they were also more likely to have a higher FIGO score (Table 1; $P<0.001$ ). Figure S1 (see Supplementary material) shows that of the 39 patients with lung metastases that became resistant to MTX/FA, 16 (41\%) subsequently received etoposide, methotrexate, actinomycin D, cyclophosphamide, vincristine (EMA/CO), and $23(59 \%)$ received dactinomycin as second-line treatment to induce remission. Similarly, of the 405 patients without metastases who developed MTX resistance $(n=379)$ or toxicity $(n=26)$, subsequent remission was induced by EMA/CO $(n=200,49.4 \%), \quad$ dactinomycin $(n=200$, $49.4 \%)$, carboplatin $(n=3,0.7 \%)$ or hysterectomy $(n=2$, $0.5 \%$ ) (see Supplementary material, Figure S2).

Disease relapse was significantly more prevalent in patients with lung metastases than in patients without metastases $(9.2 \%$ versus $2.7 \%, P=0.012)$. We postulated that the reason for the significantly increased resistance to MTX/FA and higher recurrence rate after chemotherapy in the lung metastases group might be a consequence of the increased proportion of patients with a non-molar diagnosis such as choriocarcinoma. We therefore performed subgroup analyses comparing patients who had post-molar GTN with those who had choriocarcinoma or a non-molar GTN diagnosis (Table 3). In all three subgroups, patients with lung metastases showed a significantly increased risk for developing resistance to MTX/FA. However, this was most obvious in the non-molar patients. Hence, all choriocarcinoma patients with lung metastases and all but one with non-molar GTN developed resistance to MTX/FA. However, the risk of recurrence in patients with post-molar GTN or choriocarcinoma, although higher in the lung metastases group, was no longer significant (Table 3). Importantly, disease-specific survival in all groups was $100 \%$. One patient without metastases, in remission after EMACO following MTX toxicity, died of unrelated heart failure 8.5 years after diagnosis of GTN. Median follow-up time of all the patients was 54 months (interquartile range 34.75-87.25 months).

Table S1 (see Supplementary material) shows the factors affecting MTX resistance among low-risk GTN patients, using univariate logistic regression. In the univariate analysis, four variables were found to significantly affect MTX resistance (age, interval from end of antecedent pregnancy to treatment, pre-chemotherapy hCG values and the presence of lung metastases). In the multivariate analysis, patients who had a pre-chemotherapy hCG concentration of $>100000 \mathrm{IU} / \mathrm{l}$ had a much higher risk of MTX resistance (odds ratio [OR] 13.6, 95\% CI 4.0-46.2, $P<0.001$ ) in comparison with patients whose pre-chemotherapy hCG concentration was $<1000 \mathrm{IU} / \mathrm{l}$. In addition, patients with lung metastases had a three-fold excess risk of MTX resistance compared with patients without metastases (OR 3.1, 95\% CI 1.7-5.7, $P<0.001)$. Moreover, lung metastases were significantly associated with recurrence of disease in univariable

Table 3. Subgroup analysis of the histopathological diagnosis post-molar GTN and choriocarcinoma

\begin{tabular}{|c|c|c|c|c|c|c|c|c|c|}
\hline & $\begin{array}{l}\text { Post-molar } \\
\text { GTN with } \\
\quad \text { lung } \\
\text { metastases } \\
N=57\end{array}$ & $\begin{array}{c}\text { Post-molar GTN } \\
\text { without } \\
\text { metastases } \\
N=936\end{array}$ & $\begin{array}{c}P \text { - } \\
\text { value }\end{array}$ & $\begin{array}{l}\text { CC with } \\
\text { lung } \\
\text { metastases } \\
N=4\end{array}$ & $\begin{array}{c}\text { CC without } \\
\text { metastases } \\
N=17\end{array}$ & $\begin{array}{c}P \text { - } \\
\text { value }\end{array}$ & $\begin{array}{l}\text { GTN following } \\
\text { uncertain origin } \\
\text { of pregnancy } \\
\text { with lung } \\
\text { metastases } \\
N=4\end{array}$ & $\begin{array}{l}\text { GTN following } \\
\text { uncertain origin } \\
\text { of pregnancy } \\
\text { without } \\
\text { metastases } \\
N=22\end{array}$ & $\begin{array}{c}P \text { - } \\
\text { value }\end{array}$ \\
\hline $\begin{array}{l}\text { MTX } \\
\text { resistance }\end{array}$ & $32 / 57(56.1 \%)$ & $370 / 936(39.5 \%)$ & 0.018 & $4 / 4(100 \%)$ & $4 / 17(23.5 \%)$ & 0.012 & $3 / 4(75 \%)$ & $5 / 22(22.7 \%)$ & 0.072 \\
\hline Recurrence & $4 / 57(6.7 \%)$ & $23 / 936(2.5 \%)$ & 0.064 & $2 / 4(50 \%)$ & $3 / 17(17.6 \%)$ & 0.228 & $0 / 4(0 \%)$ & $0 / 22(0 \%)$ & N/A \\
\hline
\end{tabular}


(OR 3.1, 95\% CI 1.2-8.5, P=0.02) and multivariable (OR 3.2, 95\% CI 1.1-9.4, $P=0.04)$ logistic regression analyses, adjusted for antecedent pregnancy, histopathological diagnosis and interval from evacuation until treatment. In addition, we performed a weighted logistic regression analysis that also demonstrated the presence of lung metastases as a significant adverse factor (OR 3.2, 95\% CI 1.6-6.4, $P=0.001$ ) for recurrence of low-risk disease.

\section{Discussion}

\section{Main findings}

This study demonstrates that the presence of lung metastases upon first GTN staging is a risk factor for MTX resistance and recurrence in low-risk GTN. Furthermore, a greater number of MTX courses were needed in patients with lung metastases to achieve remission compared with patients without metastases.

\section{Strengths and limitations}

The influence of lung metastases has been recently investigated by Vree et al. ${ }^{9}$ In this study, GTN patients (including high-risk patients) with lung metastases showed a higher recurrence rate $(16.7 \%$ versus $2.2 \%, P<0.0001)$ and more disease-specific deaths $(8.3 \%$ versus $0 \%, P<0.0001)$ compared with patients without metastases. The present study confirms that low-risk patients treated with MTX have higher recurrence rates if they have lung metastases present (9.2\% versus $2.7 \%, P=0.012)$. However, in contrast to the previous work, we did not validate an adverse outcome on survival in the presence of lung metastases as disease-specific survival was $100 \%$ for all low-risk patients. Our study is limited by the fact that it was a retrospective rather than a prospective analysis. Furthermore, although our study has addressed the importance of lung metastases at presentation as a potential predictor of increased risk of recurrence, we did not look at the importance of residual lung lesions following completion of chemotherapy. Such residual lesions might be expected to also increase the risk of recurrence. However, a previous study by Powles et al. failed to show any significant adverse effect on recurrence rate in low-risk GTN patients with or without residual radiological chest abnormalities following chemotherapy. ${ }^{16}$ This study was small, with 76 patients in total of whom only 23 had residual lung lesions visible on CXR and/or CT. Given the present findings, it seems sensible to perform larger studies looking at the effect of lung lesions at presentation and on completion of therapy for their effect on recurrence rates for GTN. Finally, a time-to-event analysis was not used but data were analysed as a binary (yes/no) outcome instead. The results from this method are valid under the assumption that all patients have been followed for the complete course of the disease.
Despite these limitations, we included a large homogeneous group of patients that were treated in a specialised centre with long-term follow up. In addition, all patients underwent centralised pathological review and imaging. As all GTN cases are centrally registered in the UK, the possibility of case ascertainment bias is exceptionally small.

\section{Interpretation}

Patients with choriocarcinoma had a higher risk of recurrence compared with patients with post-molar GTN (Table 3). This raises the question as to whether it is safe to consider choriocarcinoma with lung metastases as lowrisk disease even if that is the FIGO score result. Indeed, all four choriocarcinoma patients with lung metastases developed MTX resistance. Primary multi-agent chemotherapy such as EMA/CO would have been more appropriate for these women. Similarly, most non-molar GTN patients with lung metastases ended up requiring EMA/CO therapy. In contrast, choriocarcinoma patients without metastases in the lung or at other metastatic sites were often cured (76.5\%) with MTX/FA (Table 3) as were the post-molar cases without lung metastases. Consequently, patients with choriocarcinoma or non-molar GTN without lung metastases can reasonably start MTX/FA but those with lung metastases are best given multi-agent chemotherapy from the outset. However, further research is needed to confirm our findings in larger patient cohorts.

Higher pre-treatment hCG levels have been associated with an increased risk of initial chemotherapy resistance in low-risk GTN. ${ }^{17-19}$ This is in accordance with our findings that patients with lung metastases and higher hCG levels showed more MTX resistance (60\% versus 38.9\%; $P=0.001)$. Moreover, multivariate regression analysis demonstrated high pre-treatment hCG levels and the presence of lung metastases as significant adverse factors affecting MTX resistance (see Supplementary material, Table S1). The high hCG level reflects higher tumour burden. It is therefore not surprising that more courses of chemotherapy were needed to achieve complete remission in this group.

Previous studies have reported the effect of metastatic disease on response to single-agent chemotherapy in lowrisk disease. ${ }^{17,20-23}$ Chapman-Davis et al. found an increased rate of resistance to initial chemotherapy in lowrisk GTN patients with metastases (53 lung, 5 vagina and 1 pelvis) compared with the group without metastases (31\% versus $17 \% ; P=0.018) .{ }^{17}$ Mousavi et al. did not further specify metastatic disease, but higher risk of resistance was found among low-risk GTN patients with metastases $(\mathrm{OR}=8.42, P=0.001)$ compared with patients without metastases. ${ }^{21}$ Furthermore, Darby et al. found that low-risk GTN patients with lung metastases were more likely to need the switch to second-line chemotherapy than patients 
with non-metastatic GTN $(\mathrm{OR}=2.25, P=0.016){ }^{20}$ Additionally, the presence of lung metastases was an independent predictor of requiring additional MTX courses and alternative chemotherapy for women with low-risk GTN in the study of Growdon et al. $^{23}$ The mechanisms of chemotherapeutic resistance in GTN are still unclear. In acquired drug resistance, tumours are initially responsive, but become resistant with continued treatment. Hence smaller tumours without metastatic disease are more likely to be cured before multiple resistant cells have evolved.

Lung metastases can be detected on CXR or CT scan the latter being able to detect much smaller lesions of questionable significance. Indeed, the significance of metastases that are undetected by CXR but present on chest CT has been examined in multiple previous GTN studies with conflicting results. Some studies identified the presence of metastases seen on chest CT but not CXR as a significant prognostic factor for the development of resistance to single-agent chemotherapy ${ }^{24}$ or a need to change from firstline therapy. ${ }^{22,25}$ In contrast, other studies concluded that metastases detected by chest CT alone did not affect the clinical outcome of patients. ${ }^{20,26,27}$ For this reason, chest CT is not routinely undertaken in patients developing GTN following a molar pregnancy. In the present study, we cannot exclude that some patients included in the non-metastatic group have small lung metastases as chest CT scans are not standard procedure. Therefore, we cannot properly determine the importance of the $<1 \mathrm{~cm}$ CT lesions not seen on CXR.

It is still a matter of debate whether two or three consolidation courses have to be administered after normalisation of hCG. In the only study comparing two and three courses, ${ }^{28}$ the recurrence rate was significantly lower after three courses of single-agent chemotherapy $(4.0 \%$ versus $8.3 \%)$. However, in the group with two consolidation courses, more patients with lung metastases were present. Indeed, in the current study, $9.2 \%$ of the patients with lung metastases had a recurrence. Lung metastases may not completely explain the difference in recurrence rate, but it is tempting to speculate that this specific patient group with lung metastases may benefit from more than two courses of consolidation therapy.

\section{Conclusion}

In summary, the presence of lung metastases appears to have an adverse influence on the clinical course of disease in low-risk GTN. ${ }^{9}$ However, the prognosis of low-risk patients treated in a specialised trophoblastic disease centre is excellent, with a cure rate of $100 \%$. Choriocarcinoma patients with lung metastases are likely to become resistant to MTX and primary multi-agent chemotherapy such as
EMA/CO should be considered. The presence of lung metastases at presentation does increase the risk of MTX resistance and recurrence of disease but it is not an independent prognostic factor in the FIGO score because it is accounted for by the number of metastases and tumour size. Therefore an adjustment of current scoring systems to value lung metastases differently does not yet seem justified.

\section{Disclosure of interests}

None declared. Completed disclosure of interest forms are available to view online as supporting information.

\section{Contribution to authorship}

MF, CL and MS contributed to the study concept and design. MF, CL, DS, MS and RF contributed to the acquisition of the data. MF and EvW analysed and MF, CL, EvW, MS interpreted the data. MF, CL and MS were the primary authors of the manuscript and made substantial contributions to the interpretation of the data. CL, NvT, MtKB, LM, EvW, DS, XA, RF, BK, NS, NJS and MS critically revised the paper and all authors approved the final version.

\section{Details of ethics approval}

The local ethics review board committee chair was consulted and confirmed that as all patient identifiable data were removed this retrospective analysis did not require formal ethics approval.

\section{Funding}

None.

\section{Acknowledgements}

MJS acknowledges that he is supported by the National Institute for Health Research (NIHR) Imperial Biomedical Research Centre (BRC) and the NIHR Cancer Research UK Imperial Experimental Cancer Medicine Centre (ECMC).

\section{Supporting Information}

Additional supporting information may be found online in the Supporting Information section at the end of the article.

Fig S1. Remission rates after methotrexate treatment for patients with lung metastases.

Fig S2. Remission rates after methotrexate treatment for patients without lung metastases.

Table S1. Factors affecting methotrexate resistance among individuals with low-risk gestational trophoblastic neoplasia. 


\section{References}

1 Seckl MJ, Sebire NJ, Berkowitz RS. Gestational trophoblastic disease. Lancet 2010;376:717-29.

2 Ngan HYS, Seckl MJ, Berkowitz RS, Xiang Y, Golfier F, Sekharan PK, et al. Update on the diagnosis and management of gestational trophoblastic disease. Int J Gynaecol Obstet 2018;143(Suppl 2):7985.

3 Lurain JR, Singh DK, Schink JC. Primary treatment of metastatic high-risk gestational trophoblastic neoplasia with EMA-CO chemotherapy. J Reprod Med 2006;51:767-72.

4 McNeish IA, Strickland S, Holden L, Rustin GJ, Foskett M, Seckl MJ, et al. Low-risk persistent gestational trophoblastic disease: outcome after initial treatment with low-dose methotrexate and folinic acid from 1992 to 2000. J Clin Oncol 2002;20:1838-44.

5 Lybol C, Centen DW, Thomas CM, ten Kate-Booij MJ, Verheijen RH, Sweep FC, et al. Fatal cases of gestational trophoblastic neoplasia over four decades in the Netherlands: a retrospective cohort study. BJOG 2012;119:1465-72.

6 Ngan HY, Kohorn El, Cole LA, Kurman RJ, Kim SJ, Lurain JR, et al. Trophoblastic disease. Int J Gynaecol Obstet 2012;119(Suppl 2): S130-6.

7 Lurain JR. Gestational trophoblastic disease II: classification and management of gestational trophoblastic neoplasia. Am J Obstet Gynecol 2011;204:11-8.

8 Eysbouts YK, Massuger L, Thomas C, Ottevanger P, Short D, Harvey $\mathrm{R}$, et al. Dutch risk classification and FIGO 2000 for gestational trophoblastic neoplasia compared. Int J Gynecol Cancer 2016;26:1712-6.

9 Vree M, van Trommel N, Kenter G, Sweep F, Ten Kate-Booij M, Massuger $L$, et al. The influence of lung metastases on the clinical course of gestational trophoblastic neoplasia: a historical cohort study. BJOG 2016;123:1839-45.

10 Froeling FEM, Ramaswami R, Papanastasopoulos P, Kaur B, Sebire NJ, Short D, et al. Intensified therapies improve survival and identification of novel prognostic factors for placental-site and epithelioid trophoblastic tumours. Br J Cancer 2019;120:587-94.

11 Horowitz NS, Goldstein DP, Berkowitz RS. Placental site trophoblastic tumors and epithelioid trophoblastic tumors: biology, natural history, and treatment modalities. Gynecol Oncol 2017;144:208-14.

12 Alifrangis C, Agarwal R, Short D, Fisher RA, Sebire NJ, Harvey R, et al. EMA/CO for high-risk gestational trophoblastic neoplasia: good outcomes with induction low-dose etoposide-cisplatin and genetic analysis. J Clin Oncol 2013;31:280-6.

13 Benedet JL, Bender H, Jones H 3rd, Ngan HY, Pecorelli S. FIGO staging classifications and clinical practice guidelines in the management of gynecologic cancers. FIGO Committee on Gynecologic Oncology. Int J Gynecol Obstet 2000;70:209-62.

14 Gynaecologists RCoOa. The management of Gestational Trophoblastic Disease. RCOG Green-top Guideline 2010.
15 Sita-Lumsden A, Short D, Lindsay I, Sebire NJ, Adjogatse D, Seckl MJ, et al. Treatment outcomes for 618 women with gestational trophoblastic tumours following a molar pregnancy at the Charing Cross Hospital, 2000-2009. Br J Cancer 2012;107:1810-4.

16 Powles T, Savage P, Short D, Young A, Pappin C, Seckl MJ. Residual lung lesions after completion of chemotherapy for gestational trophoblastic neoplasia: should we operate? Br J Cancer 2006;94:51-4.

17 Chapman-Davis E, Hoekstra AV, Rademaker AW, Schink JC, Lurain JR. Treatment of nonmetastatic and metastatic low-risk gestational trophoblastic neoplasia: factors associated with resistance to singleagent methotrexate chemotherapy. Gynecol Oncol 2012;125:572-5.

18 Lawrie TA, Alazzam M, Tidy J, Hancock BW, Osborne R. First-line chemotherapy in low-risk gestational trophoblastic neoplasia. Cochrane Database Syst Rev 2016; Cd007102.

19 McGrath S, Short D, Harvey R, Schmid P, Savage PM, Seckl MJ. The management and outcome of women with post-hydatidiform mole 'low-risk' gestational trophoblastic neoplasia, but hCG levels in excess of $100000 \mathrm{IU}$ I(-1). Br J Cancer 2010;102:810-14.

20 Darby S, Jolley I, Pennington S, Hancock BW. Does chest CT matter in the staging of GTN? Gynecol Oncol 2009;112:155-60.

21 Mousavi AS, Zamani A, Khorasanizadeh F, Gilani MM, Zendehdel K. Resistance to single-agent chemotherapy and its risk factors in lowrisk gestational trophoblastic neoplasms. J Obstet Gynaecol Res 2015;41:776-83.

22 Nevin J, Silcocks P, Hancock B, Coleman R, Nakielny R, Lorigan P. Guidelines for the stratification of patients recruited to trials of therapy for low-risk gestational trophoblastic tumor. Gynecol Oncol 2000;78:92-6.

23 Growdon WB, Wolfberg AJ, Goldstein DP, Feltmate CM, Chinchilla $\mathrm{ME}$, Lieberman ES, et al. Evaluating methotrexate treatment in patients with low-risk postmolar gestational trophoblastic neoplasia. Gynecol Oncol 2009;112:353-7.

24 Mutch DG, Soper JT, Baker ME, Bandy LC, Cox EB, Clarke-Pearson $\mathrm{DL}$, et al. Role of computed axial tomography of the chest in staging patients with nonmetastatic gestational trophoblastic disease. Obstet Gynecol 1986;68:348-52.

25 Price JM, Lo C, Abdi S, Winter MC, Coleman RE, Tidy J, et al. The role of computed tomography scanning of the thorax in the initial assessment of gestational trophoblastic neoplasia. Int J Gynecol Cancer 2015;25:1731-6.

26 Gamer El, Garrett A, Goldstein DP, Berkowitz RS. Significance of chest computed tomography findings in the evaluation and treatment of persistent gestational trophoblastic neoplasia. J Reprod Med 2004;49:411-4.

27 Ngan HY, Chan FL, Au VW, Cheng DK, Ng TY, Wong LC. Clinical outcome of micrometastasis in the lung in stage IA persistent gestational trophoblastic disease. Gynecol Oncol 1998;70:192-4.

28 Lybol C, Sweep FC, Harvey R, Mitchell H, Short D, Thomas CM, et al. Relapse rates after two versus three consolidation courses of methotrexate in the treatment of low-risk gestational trophoblastic neoplasia. Gynecol Oncol 2012;125:576-9. 